\title{
IdeAs
}

Idées d'Amériques

$4 \mid 2013$

Crises et effets de crise dans les Amériques

\section{Les défis monétaires et financiers de l'après-crise en Amérique latine}

Monetary and Financial Challenges of the Post-Crisis in Latin America

Retos monetarios y financieros de la post-crisis en América latina

Desafios monetarios e financeiros da pós crise na América latina

\section{André Cartapanis}

\section{OpenEdition}

\section{Journals}

Édition électronique

URL : https://journals.openedition.org/ideas/684

DOI : 10.4000/ideas.684

ISSN : 1950-5701

Éditeur

Institut des Amériques

Référence électronique

André Cartapanis, «Les défis monétaires et financiers de l'après-crise en Amérique latine », IdeAs [En ligne], 4 | 2013, mis en ligne le 14 octobre 2013, consulté le 18 octobre 2022. URL : http:// journals.openedition.org/ideas/684; DOI : https://doi.org/10.4000/ideas.684

Ce document a été généré automatiquement le 18 octobre 2022

\section{(c) (i) (9)}

Creative Commons - Attribution - Pas d'Utilisation Commerciale - Pas de Modification 4.0 International - CC BY-NC-ND 4.0

https://creativecommons.org/licenses/by-nc-nd/4.0/ 


\title{
Les défis monétaires et financiers de l'après-crise en Amérique latine
}

\author{
Monetary and Financial Challenges of the Post-Crisis in Latin America \\ Retos monetarios y financieros de la post-crisis en América latina \\ Desafios monetarios e financeiros da pós crise na América latina
}

André Cartapanis

\section{Introduction}

1 Le continent latino-américain a longtemps été considéré comme le champ d'application privilégié de l'instabilité monétaire et des crises financières. Nul n'a donc été surpris lorsque les économies latino-américaines ont été touchées par la crise systémique de 2007-2009 et par la récession que celle-ci a provoquée. Malgré des différences significatives selon les pays, la récession de 2008-2009 s'apparente cependant, en Amérique latine, à un trou d'air, car le redémarrage de l'activité économique est apparu rapidement, dès l'été 2009. Aujourd'hui, la croissance des économies latino-américaines a retrouvé le rythme d'avant-crise, avec plus de $6 \%$ en 2010, et encore 4,7\% en 2011 selon les prévisions du FMI publiées en avril 2011, alors que l'activité économique des pays les plus développés reste nettement plus faible et paraît hypothéquée par l'ajustement budgétaire en cours, surtout en Europe. En un mot, tout comme l'Asie, le continent latino-américain est aujourd'hui considéré comme l'un des plus solides de l'économie mondiale.

2 Est-ce dire que les économies latino-américaines ne sont pas exposées à des fragilités financières ou monétaires de nature à compromettre cette situation ? Il serait excessif de le croire compte tenu d'un ensemble de défis, sur le plan financier et en matière de pilotage macroéconomique, liés notamment aux effets de l'afflux de capitaux et aux tensions que cela occasionne sur le plan monétaire ou sur les marchés d'actifs financiers. 
3 Tel est l'objet de cette analyse que d'examiner les principaux caractères de la répercussion de la crise en Amérique latine, avant de repérer les sources de fragilité apparues dans la période d'après-crise financière et d'analyser les politiques, notamment dans le domaine macroprudentiel, que mettent en œuvre les gouvernements latino-américains afin de répondre aux menaces qui pèsent aujourd'hui sur la croissance et la stabilité financière.

\section{Les économies latino-américaines et la sortie de crise}

4 La crise des systèmes financiers qui se déclenche aux États-Unis et en Europe, en août 2007, et s'exacerbe après la faillite de Lehman Brothers, le 15 septembre 2008, a provoqué une récession de vaste ampleur qui a contaminé les pays émergents, comme l'illustrent les graphiques 1 et 2. Il apparaît également que les économies latinoaméricaines, si elles n'ont pas échappé à cette lame de fond, ont été nettement moins affectées que dans le passé. Jusqu'ici, les crises financières globales touchaient plus que proportionnellement le continent latino-américain. La crise de 2007-2009 déroge à ce principe dans la mesure où la récession épouse exactement les tendances de l'économie mondiale, ni plus ni moins. Cette répercussion réelle de la crise financière a répondu à tout un ensemble de canaux de transmission: les effets de la chute du commerce mondial et des flux touristiques, le recul des rapatriements de revenus de la part des travailleurs ayant émigré aux États-Unis ou au Canada, le contrecoup de la baisse des cours de matières premières à compter de l'automne 2008 (graphique 3). Mais à cette occasion, nulle trace, en Amérique latine, de faillites bancaires ou de défauts souverains, contrairement à ce que l'on observait aux États-Unis ou en Europe.

En fait, le choc de la récession mondiale a été quelque peu atténué dans plusieurs pays latino-américains par la mise en œuvre de politiques budgétaires contracycliques qui se sont révélées efficaces, le creusement des déficits étant toutefois sans commune mesure avec ce qui a été observé aux États-Unis ou en Europe. Mais il faut souligner que ces pays avaient réalisé des efforts d'ajustement considérables dans la période d'avantcrise, depuis 2003, et avaient amplement bénéficié de la très forte hausse des cours des matières premières, cette occurrence leur ayant permis de se désendetter ou d'accumuler des réserves de change.

6 Désormais, parmi les grandes régions de l'économie mondiale, le continent latinoaméricain doit être considéré comme l'une des zones les plus solides de la planète en matière de perspectives de croissance. La reprise est forte et l'on retrouve les taux de croissance particulièrement élevés qui avaient été enregistrés depuis 2003, la tendance étant alors de 4 à $5 \%$. Cela s'explique, pour une part essentielle, par des facteurs internes, le rebond de l'investissement et, surtout, de la consommation, mais aussi par des facteurs externes. La hausse des cours sur les marchés des matières premières, après la baisse initiée au moment de la crise, a particulièrement profité aux économies exportatrices de matières premières:le pétrole pour le Brésil, la Colombie et le Mexique; les métaux au Chili et au Pérou;les produits agricoles, alimentaires ou industriels, en Argentine et au Brésil.

7 Pour autant, la zone reste hétérogène et de fortes disparités perdurent : en 2011, le Venezuela est à la traine sur le plan de la croissance, malgré la dépréciation de la monnaie nationale ; il en est de même dans les économies de la Caraïbe ; l'inflation est préoccupante en Argentine, au Paraguay, au Venezuela, à Trinidad et Tobago... Plus 
généralement, les signes avant-coureurs d'une surchauffe économique se multiplient sur le continent latino-américain, ce dont témoigne l'existence d'un output gap positif, signe d'une croissance plus élevée que la croissance d'équilibre. C'est ce que l'on peut observer à partir des graphiques 4 et 5 .

Graphique 1. Comparaison des effets de la crise sur la croissance du PIB réel, à l'échelle mondiale, parmi les pays industriels et dans les économies émergentes

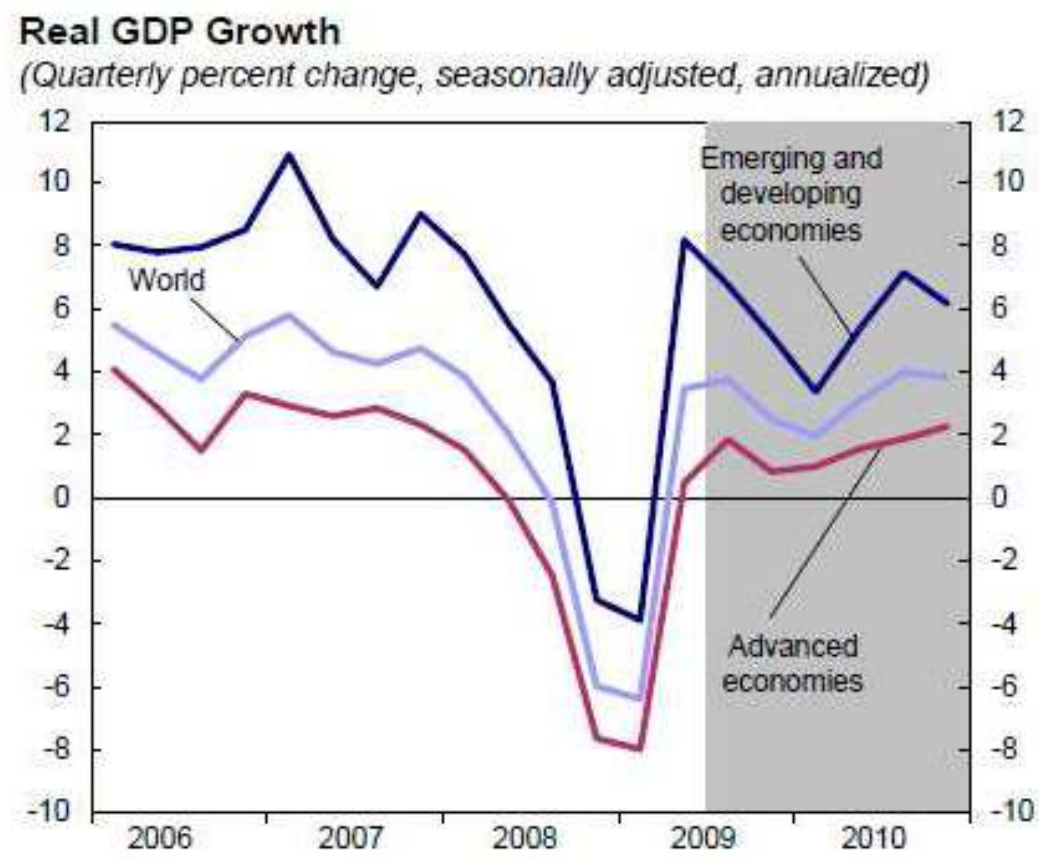

Source: IMF staff calculations.

Graphique 2. Une crise dont les effets spécifiques sur les économies latino-américaines (LAC) s'avèrent bien plus faibles que dans le passé : comparaison des effets sur la croissance du PIB lors des précédentes crises et à l'occasion de la crise de 2008-2009

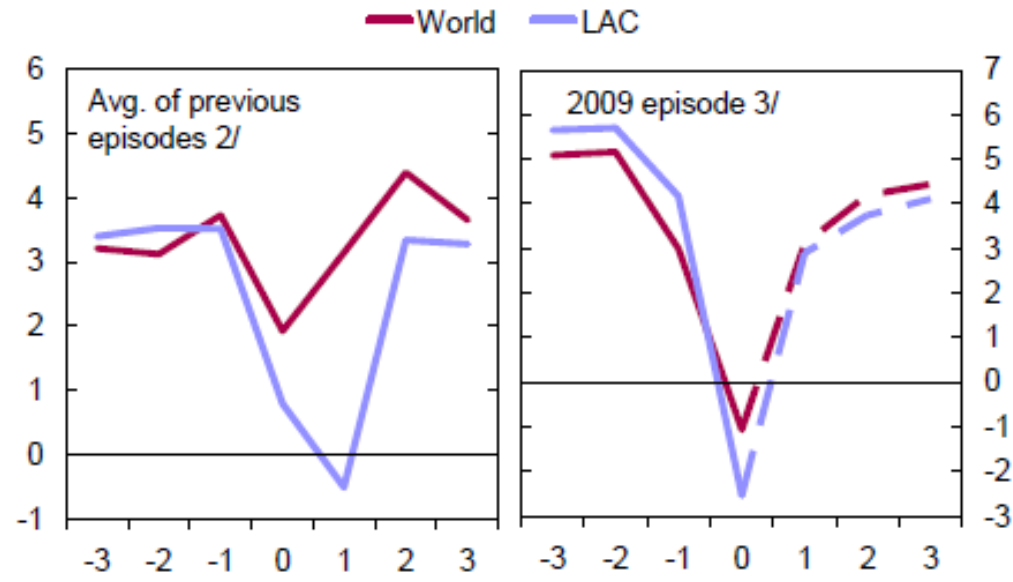


1/ GDP-PPP weighted average.

$2 /$ The data show the average evolution 3 years before and after the previous crisis episodes ( $\mathrm{t}=0$ corresponds to the episodes of years 1982,1998 , and 2001). $3 /$ The data show the evolution 3 years before and after the 2009 crisis episode $(t=0)$. Dotted lines correspond to projections.

(Source : IMF, 2009)

Graphique 3. Les facteurs de diffusion de la crise vers les économies latino-américaines

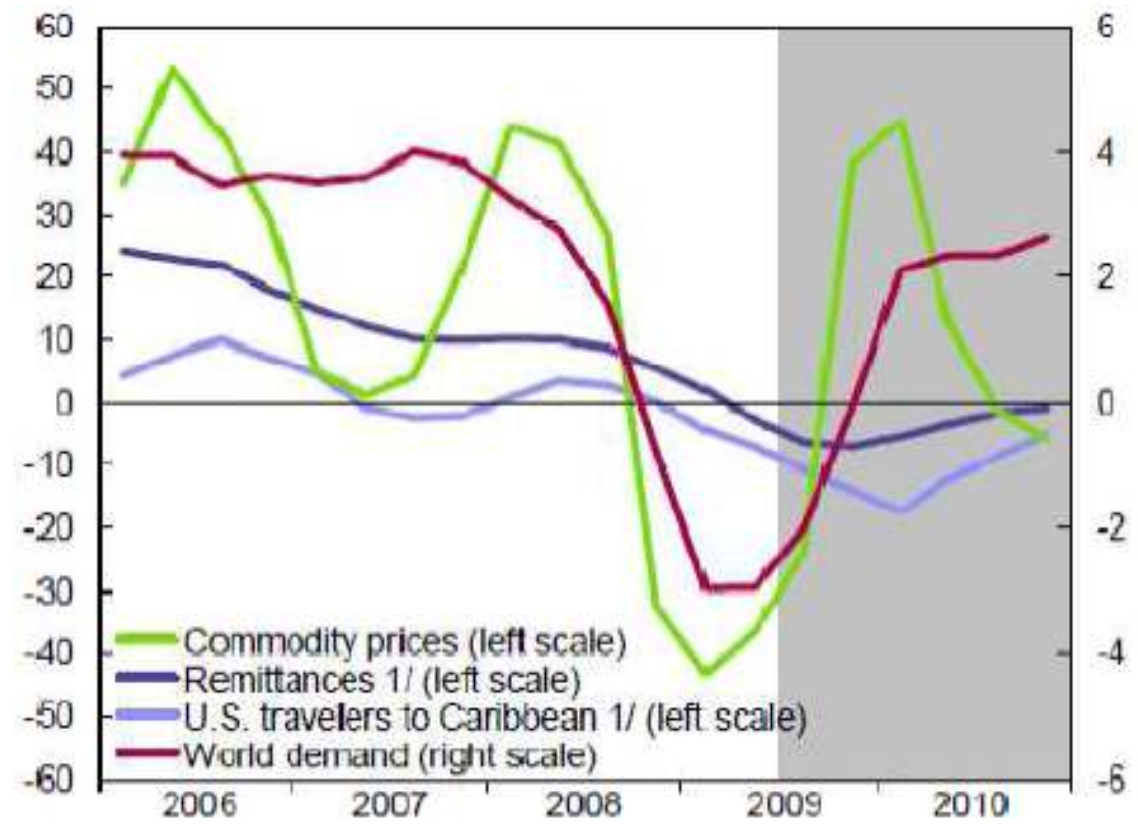

Source: IMF staff calculations.

1/Remittances and tourism are four quarter moving averages.

(Source : IMF, 2009)

Graphiques 4. La situation macroéconomique des principales économies latinoaméricaines : croissance, chômage, soldes des paiements courants, inflation

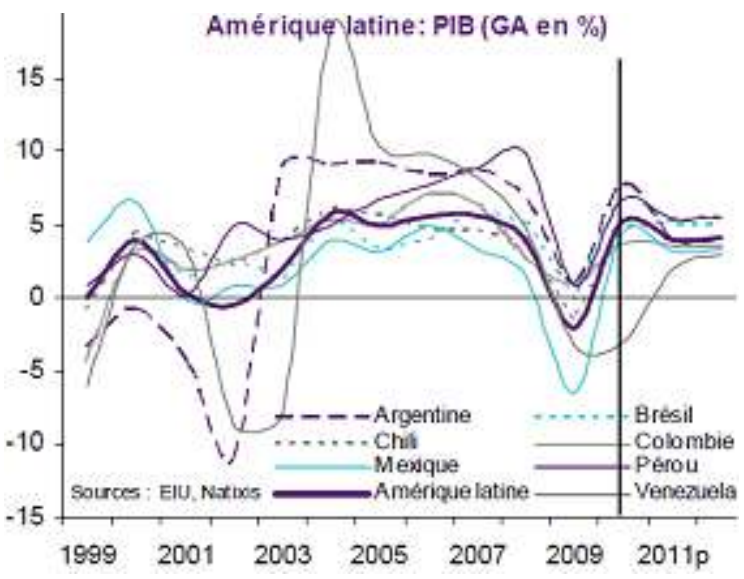

(Source : NATIXIS, 2010) 


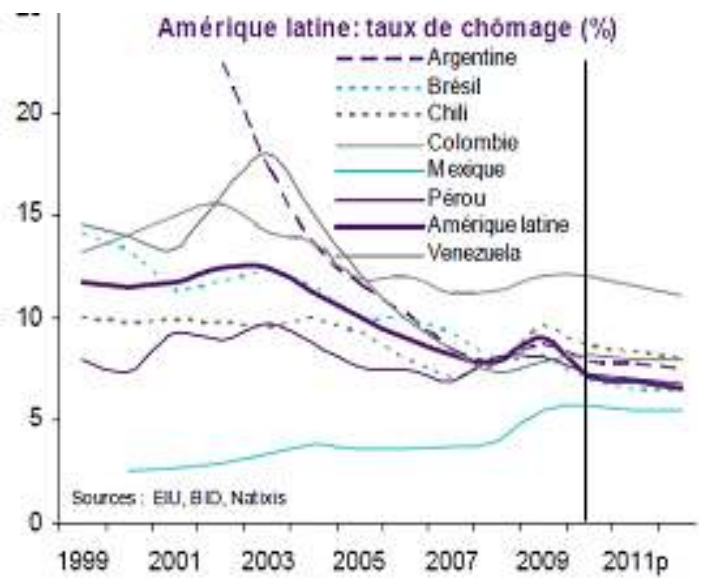

(Source: NATIXIS, 2010)

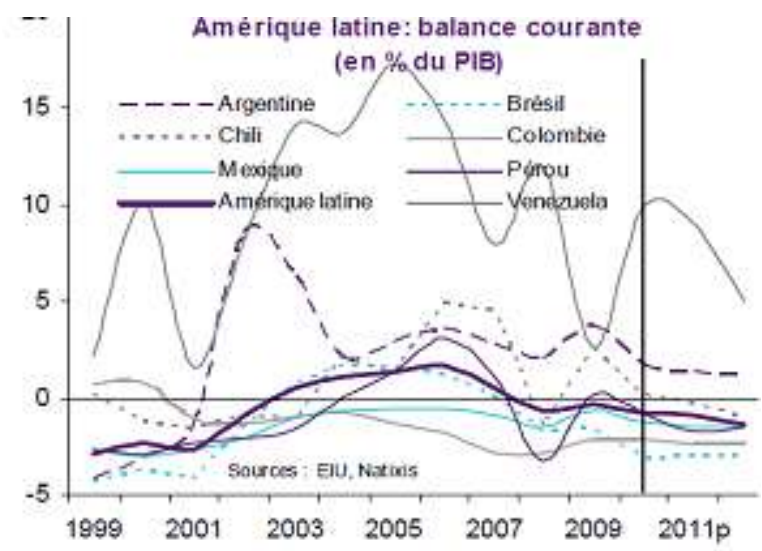

(Source : NATIXIS, 2010)

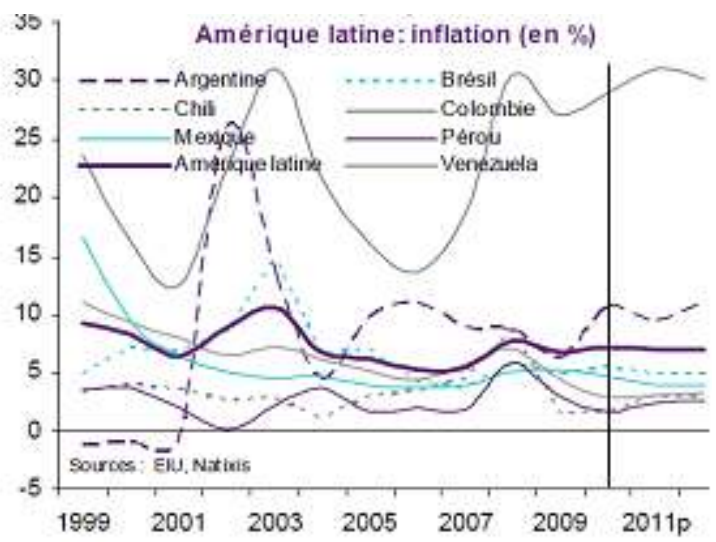

(Source : NATIXIS, 2010) 


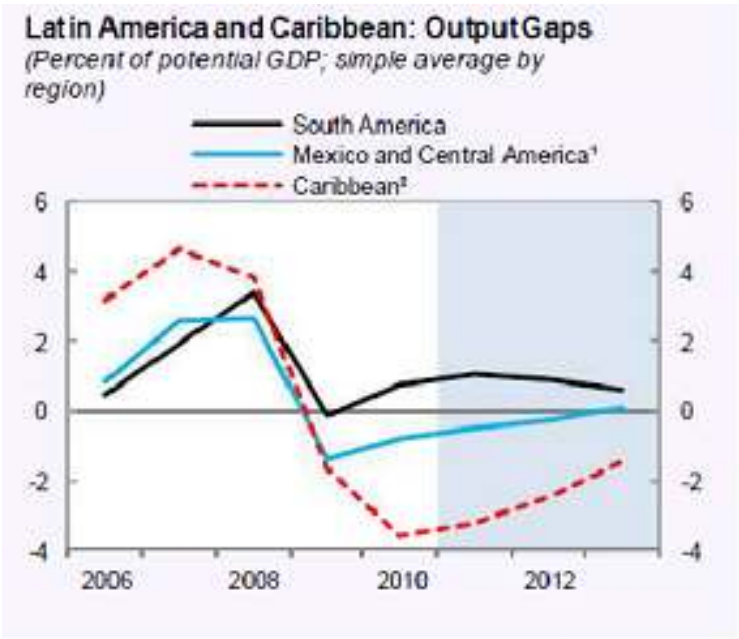

(Source : IMF, 2011)

Si l'on compare cette configuration macroéconomique à la situation des économies occidentales, l'hypothèse du trou d'air provoqué par la crise se trouve amplement confortée dans le cas de l'Amérique latine : globalement, malgré certaines spécificités nationales, la croissance est désormais élevée et les taux de chômage ont retrouvé la tendance à la baisse amorcée depuis 2003 ; les taux d'inflation avoisinent leurs niveaux d'avant-crise et les déséquilibres courants paraissent soutenables. Pour autant, en 2011, un ensemble de menaces subsiste sur le plan de la stabilité financière et monétaire.

\section{Les défis de l'après-crise financière}

Après la faillite de Lehman Brothers, dans la panique générale qui s'est emparée des marchés, d'importantes sorties de capitaux avaient été observées en provenance des pays émergents, compte tenu du climat de défiance généralisée qui avait touché les marchés de capitaux, les placements en dollars étant considérés comme des valeurs refuges. Mais depuis la fin de la récession, à compter de l'été 2009, on a assisté au retour des flux internationaux de capitaux vers les économies émergentes, non seulement en Asie, mais également parmi plusieurs économies latino-américaines, le Brésil en particulier. Cela a provoqué une appréciation significative des taux de change nominaux et, compte tenu des tentatives menées par les banques centrales de limiter cette hausse, cela a suscité également une accumulation de réserves de change en devises et une forte expansion de la liquidité globale, favorisant une accélération de la dynamique du crédit.

Aujourd'hui, la principale menace pesant sur la croissance et la stabilité financière résulte du retour des capitaux étrangers, au Brésil, au Chili, en Colombie, car ces transferts d'épargne hypothèquent la maîtrise de l'inflation, créent des tensions très fortes sur les taux de change et, surtout, contribuent à une expansion très rapide du crédit. Cela crée une situation de dilemme pour la politique macroéconomique. Une hausse des taux d'intérêt, de nature à modérer les tensions et à freiner le crédit, a toutes les chances de conforter l'attractivité de ces pays et d'amplifier les entrées de capitaux, les IDE certes, mais aussi les investissements de portefeuilles, favorisant ainsi 
la hausse des prix d'actifs, sur les marchés d'actions tout particulièrement. D'autant que ces pays bénéficient d'une très forte baisse des primes de risques sur le marché des financements corporate ou des emprunts souverains, couplée au maintien de taux d'intérêt relativement faibles parmi les pays développés, enlisés dans les difficultés de sortie de crise et les politiques de réduction des déficits budgétaires. D'où le regain d'intérêt porté aux politiques de contrôle des entrées de capitaux que le Brésil a d'ores et déjà expérimentées, nous y reviendrons. Dans le cas du Brésil, les investissements de portefeuille, qui avaient chuté à l'automne 2008, ont retrouvé une tendance ascendante très marquée, provoquant un rebond des prix d'actifs et une forte appréciation du taux de change à compter de janvier 2009. Mais cela concerne également les autres économies latino-américaines intégrées dans le système financier mondial - le Chili, la Colombie, le Mexique et, à un degré moindre, le Pérou - qui, toutes, ont vu leur monnaie s'apprécier, corrélativement à la hausse des cours boursiers et aux entrées de capitaux sous la forme d'IDE ou d'investissements de portefeuilles. C'est ce que l'on peut observer à partir des graphiques 6 et 7.

Graphique 6. Le retour des entrées de capitaux au Brésil et les effets induits sur le taux de change à compter de l'été 2009

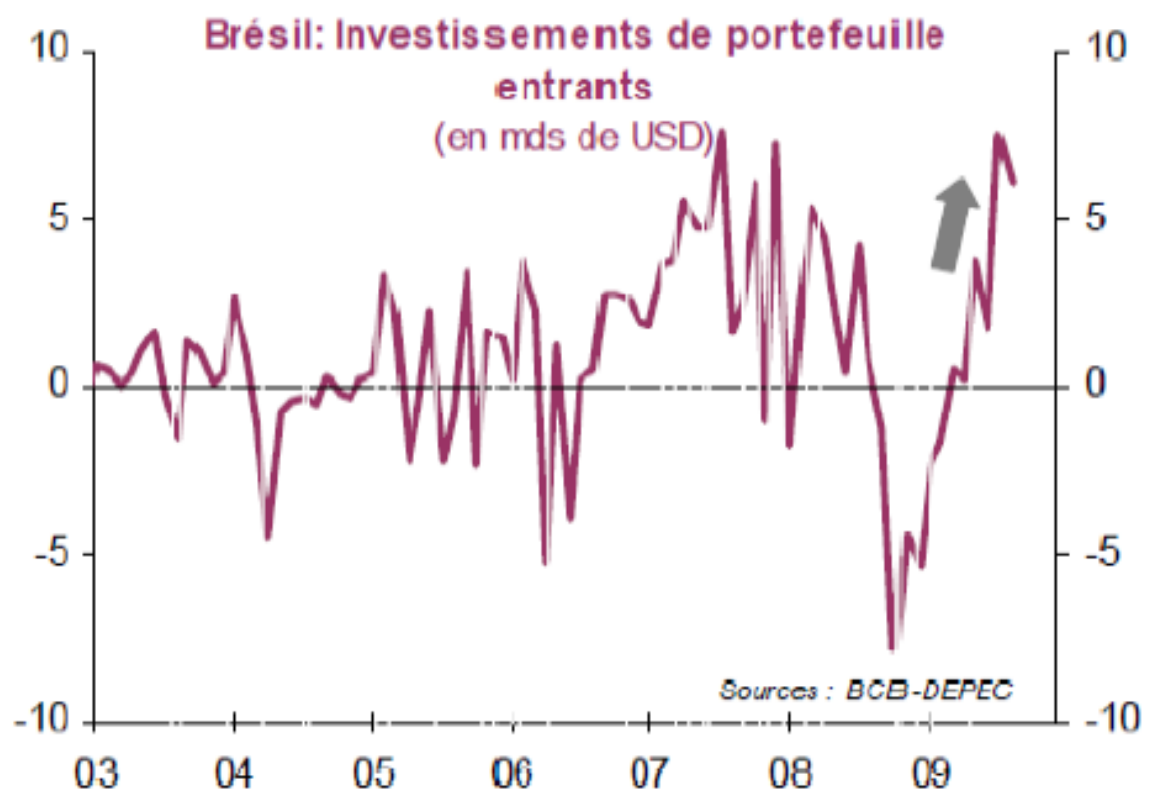

(Source : NATIXIS, 2010) 


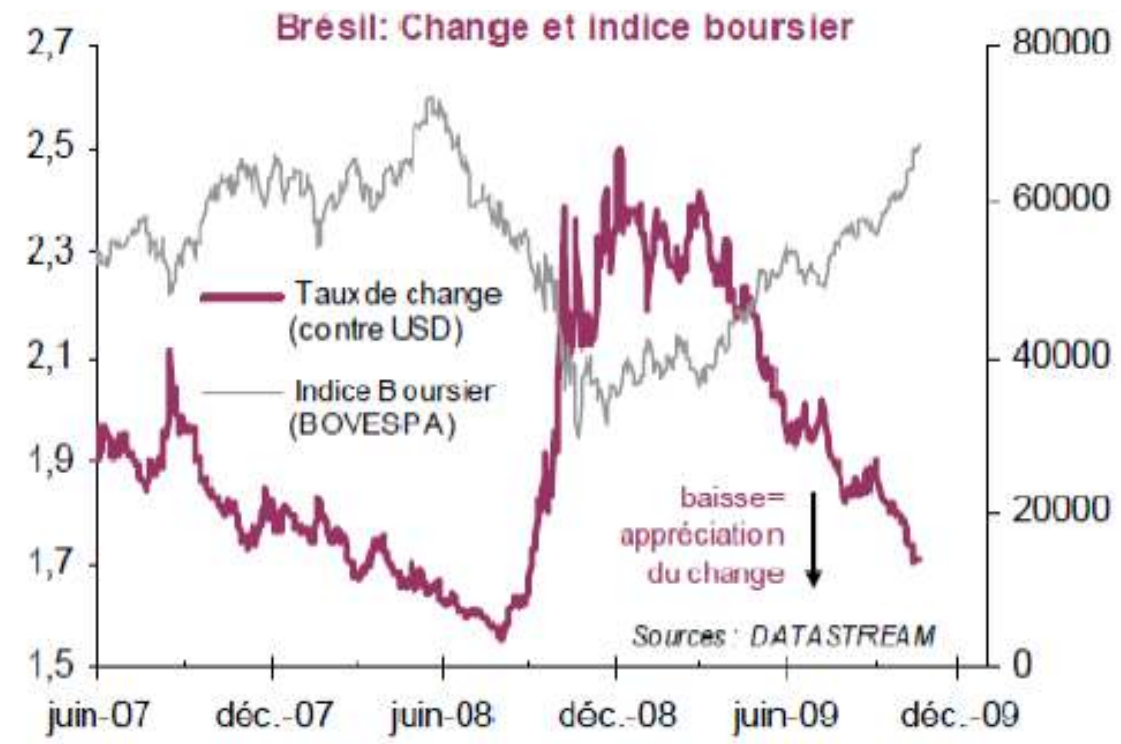

(Source : NATIXIS, 2010)

Graphiques 7. La réapparition des tensions financières en Amérique latine dès la fin de la récession : entrées de capitaux et rebond des cours boursiers

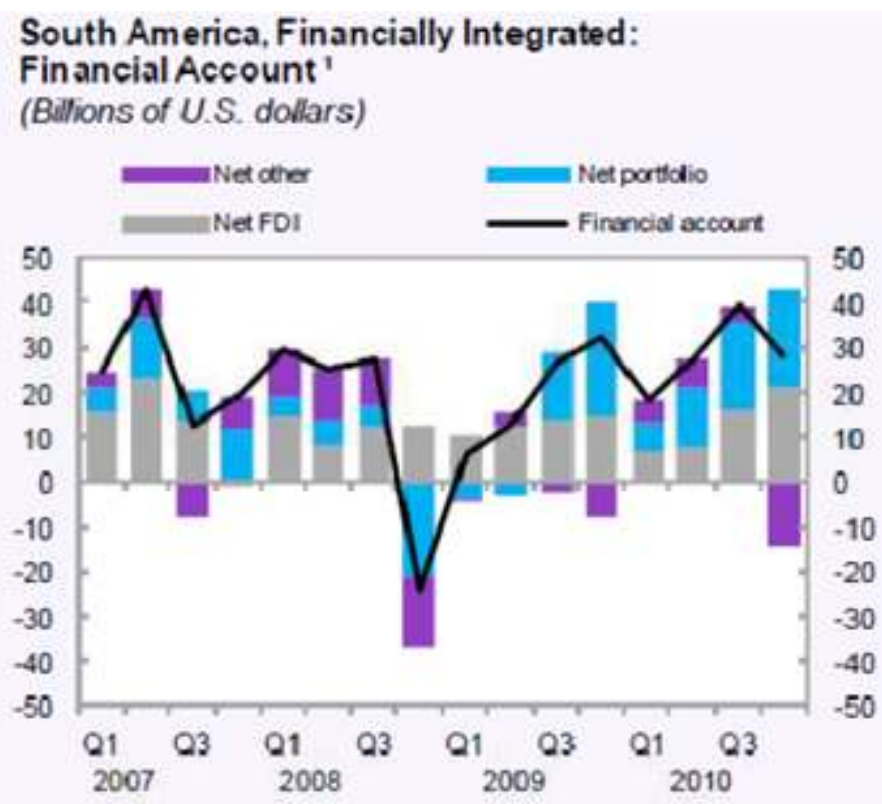

'Includes Brazil, Chile, Colombia, Peru, and Unuguay.

(Source : IMF, 2011) 


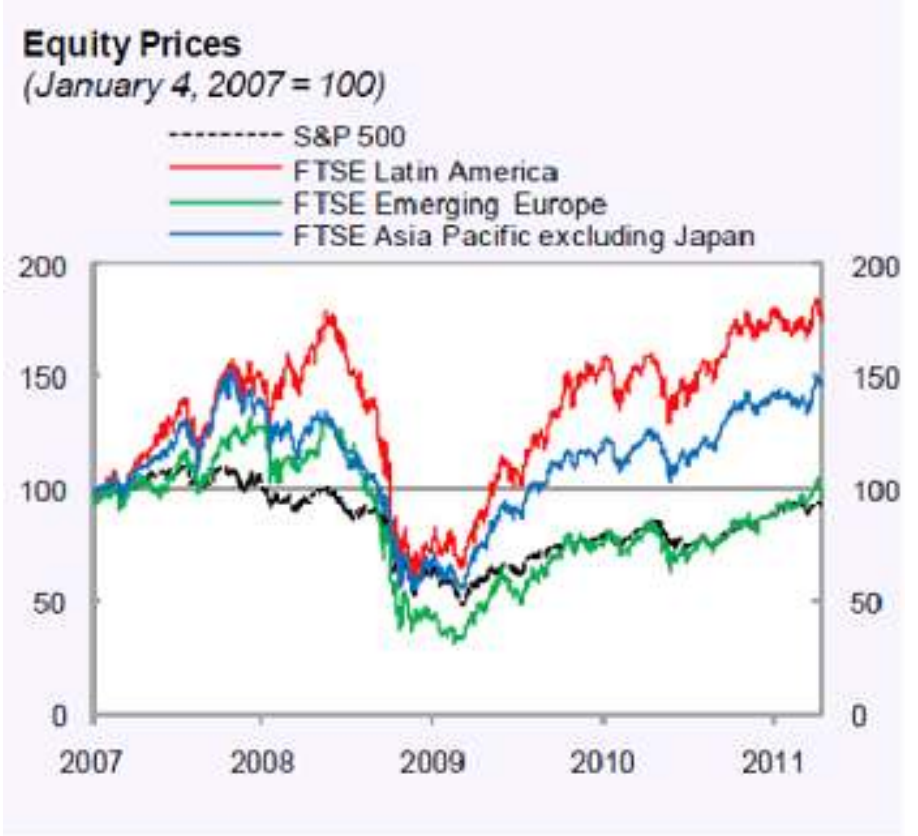

(Source : IMF, 2011)

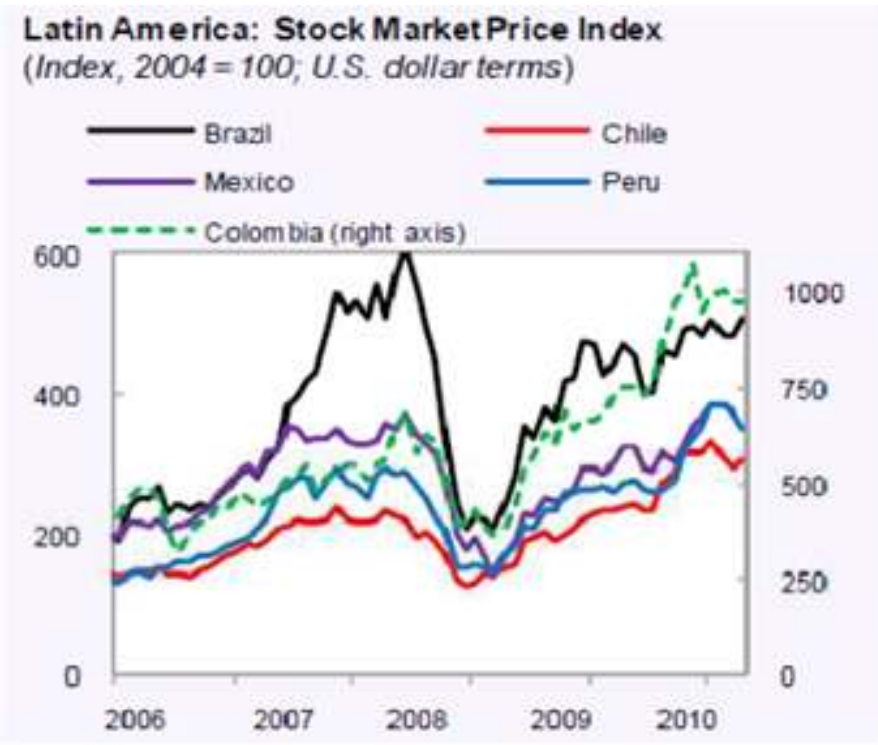

(Source : IMF, 2011)

11 Beaucoup considèrent que la flexibilité des taux de change constitue une réponse efficace face à de telles tensions associées aux entrées de capitaux. Mais l'appréciation qui en résulte pourrait bien conduire les taux de change nominaux à des niveaux insoutenables en mettant en cause la compétitivité des secteurs exportateurs. La solution des politiques d'intervention des banques centrales sur le marché des changes est souvent invoquée. Mais au delà du fait que leur efficacité n'est jamais garantie, surtout sur une base unilatérale, les conséquences sur la masse monétaire restent difficiles à maîtriser et tout porte à penser qu'elles contribueraient à alimenter la liquidité globale et, donc, à nouveau, la dynamique du crédit.

Toute la question est donc de savoir jusqu'où cette configuration macroéconomique est soutenable, notamment sous l'angle de la stabilité financière. Car le risque existe d'une 
crise financière de second tour, sorte d'effet collatéral de la sortie de crise, si le boom s'accentue.

13 Le problème que rencontrent aujourd'hui les pays latino-américains ne leur est pas spécifique et concerne en fait la dimension macroéconomique de la globalisation financière. Les politiques de libéralisation financière ont été très largement justifiées par les avantages d'une circulation plus large de l'épargne mondiale permettant de financer l'investissement et la croissance parmi les économies émergentes. Mais on a sous-estimé les contraintes macroéconomiques induites par l'afflux de capitaux, en Asie comme sur le continent latino-américain. L'excès de liquidités externes peut exiger un durcissement de la politique monétaire et une hausse des taux d'intérêt réels. Les entrées de capitaux peuvent engendrer une appréciation du taux de change réel, de nature à freiner le rythme de développement dans le secteur des biens échangeables.

C'est exactement la situation dans laquelle se trouvent bon nombre de pays latinoaméricains depuis 2010, soumis au risque d'appréciation du taux de change réel que suscite le rebond des entrées de capitaux et à ses effets sur la profitabilité du secteur des biens échangeables et sur le niveau d'investissement dans le secteur exposé à la compétition internationale, le plus important pour la croissance. Car la hausse du taux de change réel réduit la profitabilité relative de l'industrie et ralentit la croissance globale. On ne doit pas non plus oublier le risque d'une dynamique explosive de la dette externe, source d'une montée des fragilités financières et de nature à interrompre brutalement ces transferts d'épargne, avant de déclencher une crise : c'est l'hypothèse du sudden stop, popularisée par Guillermo Calvo.

Cette configuration se double d'un risque de surchauffe, sur le plan macroéconomique interne, compte tenu de la dynamique du crédit. Or, depuis la sortie de crise, le volume du crédit a retrouvé une tendance extrêmement rapide, de l'ordre de 10 à $15 \%$ par an, surtout parmi les pays latino-américains les plus intégrés à la finance mondiale (Brésil, Chili, Colombie, Mexique, Pérou et Uruguay), alimenté par les entrées de capitaux, ce qui contribue au regain des tensions inflationnistes et provoque de nouvelles bulles, on l'a vu sur les marchés boursiers, mais aussi dans l'immobilier. C'est ce que montrent très clairement les Graphiques 8.

Notons ici que de nombreux pays latino-américains ont choisi de mener une politique monétaire explicite de ciblage de l'inflation, et c'est le cas du plus important d'entre eux, le Brésil.

Un consensus semble s'être dégagé parmi les économistes pour dire que dans les pays où des banques centrales indépendantes pratiquent l'inflation targeting, on ne rencontre pas de difficultés particulières dans le contrôle de l'inflation. En général, ces pays ont libéralisé leurs balances des capitaux, laissent leur taux de change flotter, sans variabilité excessive du change. Ils n'enregistrent plus de crises de balance des paiements et ne semblent pas rencontrer de problèmes particuliers avec leur balance courante. Cette configuration, où les banques centrales contrôlent l'inflation domestique sans se soucier des évolutions extérieures, des taux de change notamment, serait donc efficace sur le plan de la stabilité macroéconomique.

Mais on peut avancer aussi la thèse inverse:une dynamique instable peut se déclencher si la banque centrale mène une politique active de hausse des taux d'intérêt en réaction à l'inflation. Les flux de capitaux jouent alors un rôle procyclique, potentiellement déstabilisant. Si la croissance est forte, les capitaux affluent et stimulent l'économie à court-terme. Mais cela s'opère au prix d'un accroissement des 
niveaux d'endettement et, surtout, cela favorise la surévaluation réelle du change. Cette politique a toutes les chances de conduire à une hausse du taux d'intérêt à long terme qui, indépendamment des effets sur la demande d'investissement et la croissance, fait diverger la dette, aussi bien domestique qu'extérieure. La progression rapide de la dette peut même conduire à des anticipations de défaut des emprunteurs, à un arrêt des entrées de capitaux, susceptibles de déclencher une crise de liquidité bancaire qui ne saurait épargner les emprunteurs domestiques.

Tel est le scénario qui conduit certains économistes à recommander le recours à des contrôles sur les entrées de capitaux et telle est la configuration macroéconomique à risque de l'Amérique latine en 2011. Quelles sont alors les politiques économiques que peuvent engager les gouvernements et les banques centrales en Amérique latine pour maittriser ces sources de tensions sans interrompre la reprise?

Graphiques 8. L'accélération de la dynamique du crédit dans les pays latino-américains en période d'après-crise
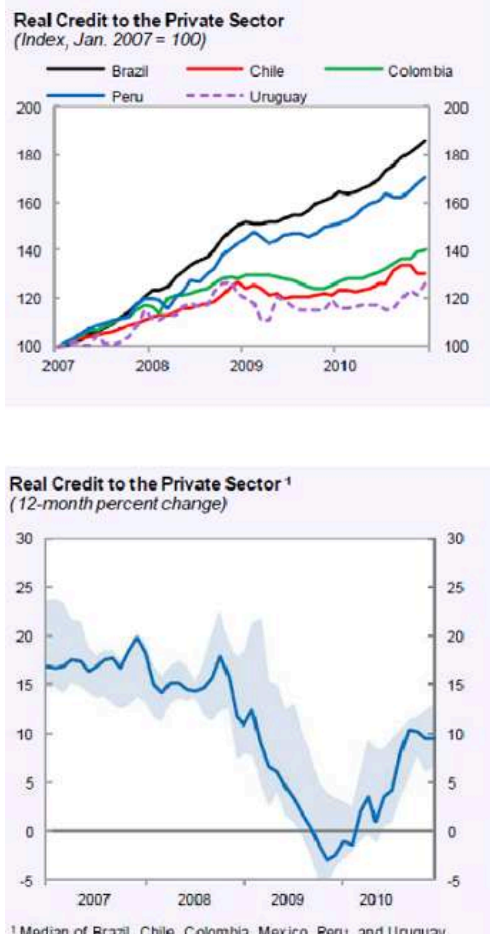

IMedian of Brazs, Chile, Colombia, Mexico, Peru, and Uruguay.
Shaded area is the 25 th to 75 th percentile range,

(Source : IMF, 2011)

\section{Le recours aux politiques macroprudentielles pour maîtriser les sources d'instabilité financière sans mettre en cause la reprise en Amérique Latine}

Aucune politique simple ne paraît capable de neutraliser ou, à tout le moins, d'atténuer de tels défis. Tous les instruments paraissent devoir être mobilisés : une politique monétaire modérément plus restrictive; grâce aux interventions sur le marché des changes, une appréciation maîtrisée du taux de change ; et, cela, sans infléchissement marqué sur le plan budgétaire compte tenu des difficultés politiques qu'un ajustement 
drastique pourrait induire. Les contrôles sur les entrées de capitaux peuvent également s'imposer, d'où la décision du Brésil, le 19 octobre 2009, d'instaurer une taxe sur les investissements de portefeuilles (actions ou obligations) de $2 \%$. Mais la solution la plus innovante consiste à mettre en œuvre une politique macroprudentielle contracyclique.

Si elle vise la prévention de l'ensemble des risques associés aux activités financières des banques, la réglementation prudentielle répond à deux objectifs distincts. Elle doit contribuer à la sécurité de chaque intermédiaire bancaire afin de protéger les déposants ou les investisseurs face à d'éventuelles défaillances individuelles. Telle est la fonction visée par les dispositifs prudentiels traditionnels, comme Bâle II. Mais la réglementation bancaire peut également stabiliser le système monétaire et financier dans sa dimension globale, en limitant le risque d'un boom du crédit susceptible d'induire, à terme, une détresse financière et des pertes significatives en termes d'output réel.

Mais comment atténuer la procyclicité, tout à la fois du crédit et de la prise de risque des banques? Le plus simple est d'agir sur le levier net du système financier au cours $\mathrm{du}$ cycle $\mathrm{du}$ crédit. On peut tenter également d'introduire la dynamique macroéconomique dans la quantification du risque de crédit en considérant que l'évolution prévisible du cycle des affaires, mais également celle des prix d'actifs, en présence d'une bulle par exemple, rétroagiront nécessairement sur la qualité future des créances, ce que les méthodes usuelles négligent. On peut ainsi procéder à un renforcement discrétionnaire des provisions minimales pour risques dans les périodes de haute conjoncture, alors même que les institutions financières ont tendance à les alléger et que les agences de notation, de la même manière, s'avèrent peu sensibles à la cyclicité de l'activité tant que celle-ci reste soutenue. Telle est également la finalité d'un mécanisme de provisionnement dynamique reliant les normes prudentielles aux variations, et non pas au niveau, des créances bancaires ou des prix d'actifs.

Parmi tous ces dispositifs, le plus emblématique est constitué par la mise en place d'exigences réglementaires en capitaux propres, non pas en fonction de la situation spécifique d'un établissement, mais en réponse à la situation cyclique de l'économie. Ces volants de fonds propres contracycliques doivent être adossés à un proxy du risque systémique, par exemple l'écart du ratio crédit/PIB vis-à-vis de sa tendance, afin qu'ils augmentent en phase d'expansion et qu'ils diminuent en phase de contraction. On peut également introduire un ratio de levier pour limiter les crédits à risque en phase de boom.

24 Le Comité de Bâle sur la supervision bancaire a préparé un package de nouveaux standards internationaux, dénommé Bâle III, incluant ces nouvelles dispositions macroprudentielles. Bâle III a été finalisé en septembre 2010 et a été approuvé par le G 20 de Séoul.

Conçus afin de limiter le risque d'un nouvel accident systémique au sein des pays industriels, ces dispositifs paraissent particulièrement adaptés à la situation des économies latino-américaines et aux nombreux dilemmes qui contraignent la politique macroéconomique en présence d'importantes entrées de capitaux. C'est là un moyen privilégié de contrecarrer le boom du crédit et la surréaction des prix d'actifs qui pourraient bien, à un moment ou un autre, provoquer un mouvement de défiance et interrompre la croissance. Comme l'ont fait auparavant les autorités monétaires à Hong-Kong ou en Corée, les gouvernements latino-américains ont donc amorcé l'application de tels instruments macroprudentiels. Le Brésil et le Pérou, parmi les 
premiers, en octobre 2010, mais également la Bolivie et la Colombie, ont adopté un renforcement des exigences contracycliques en capitaux propres et ont exigé des provisionnements exceptionnels sur les positions externes en dollars et à court terme des banques. L'enjeu est de taille car la combinaison d'une situation de surchauffe sur le plan macroéconomique et de bulles sur les marchés d'actifs en réponse à la dynamique du crédit augmente considérablement le risque d'une nouvelle crise financière en Amérique latine.

\section{BIBLIOGRAPHIE}

Cartapanis, André, (en coll. avec Patrick Artus), « Globalisation financière et croissance dans les économies émergentes. La sous-estimation des contraintes macroéconomiques induites ", Revue Economique, vol. 59, $\mathrm{n}^{\circ}$ 6, novembre 2008, p. 1145-1158.

Cartapanis, André, « La crise financière et les politiques macroprudentielles : inflexion réglementaire ou nouveau paradigme? ", Revue Economique, vol. 62, n 3, mai 2011, p. 349-382.

ECLAC, Economic Survey of Latin America and the Caribbean. The distributive impact of public policies, 2009-2010, ECLAC, United Nations, 2010.

IMF, Western Hemisphere. Crisis Averted. What's Next?, Regional Economic Outlook, IMF, Octobre 2009.

IMF, Western Hemisphere. Watching Out for Overheating, Regional Economic Outlook, IMF, Avril 2011.

NATIXIS, « Un panorama des grandes régions de l'économie mondiale, trois ans après le déclenchement de la crise », Flash Economie, n 476, 22 septembre 2010.

\section{RÉSUMÉS}

La crise financière internationale de 2007-2009 n'a pas épargné le continent latino-américain. Mais la récession a été moins grave et surtout de plus courte durée que lors des crises précédentes. Toutefois, dans un contexte de croissance élevée et de résurgence des tensions inflationnistes, le retour des entrées de capitaux alimente une dynamique accélérée du crédit qui provoque de nouvelles bulles sur les marchés d'actifs (taux de change, marchés boursiers) et laisse planer le risque d'une nouvelle crise financière. Les gouvernements latino-américains doivent donc affronter de nouveaux défis monétaires et financiers dans cette période d'aprèscrise en essayant de maîtriser les tensions financières sans brider la croissance.

The global financial crisis of 2007-2009 has not spared the Latin American continent. But the recession has been less severe, and especially shorter than in previous crises. However, in a context of high growth and resurgence of inflationary pressures, the return of capital inflows fueling an accelerated credit dynamics that cause new bubbles in asset markets (exchange rates, equity markets) and casts risk of another financial crisis. Latin American governments must face new monetary and financial challenges in the post-crisis period trying to master the financial stress without limiting growth. 
La crisis financiera mundial de 2007-2009 no ha escatimado en el continente latinoamericano. Pero la recesión ha sido menos grave, y sobre todo más corta que en crisis anteriores. Sin embargo, en un contexto de alto crecimiento y el resurgimiento de las presiones inflacionarias, el retorno de los flujos de capital impulsando una dinámica acelerada de crédito que causan nuevas burbujas en los mercados de activos (tipos de cambio, los mercados de renta variable) y pone riesgo de una nueva crisis financiera. Los gobiernos latinoamericanos deben enfrentar nuevos retos monetarios y financieros en el período posterior a la crisis tratando de dominar el estrés financiero sin limitar el crecimiento.

A crise financeira mundial de 2007-2009 não poupou o continente latino-americano. Mas a recessão foi menos severa, e, especialmente, mais curta do que em crises anteriores. No entanto, num contexto de elevado crescimento e ressurgimento de pressões inflacionárias, o retorno dos fluxos de capitais alimentando uma dinâmica acelerada de crédito que fazem com que novas bolhas nos mercados de ativos (taxas de câmbio, os mercados accionistas) e lança risco de outra crise financeira. Governos latino-americanos têm de enfrentar novos desafios monetários e financeiros no período pós-crise, tentando dominar o estresse financeiro sem limitar o crescimento.

\section{INDEX}

Mots-clés : crise financière, mouvements internationaux de capitaux, inflation, bulles sur les marchés d'actifs, contrôles des mouvements de capitaux, politique économique, économies d'Amérique latine

Palavras-chave : crise financeira, movimentos internacionais de capitais, inflação, bolhas nos mercados de ativos, controle dos movimentos de capitais, política econômica, economias da América Latina

Palabras claves : crisis financiera, los movimientos internacionales de capital, inflación, las burbujas en los mercados de activos, control de los movimientos de capital, política económica, las economías de América Latina

Keywords : financial crisis, international capital movements, inflation, asset markets bubbles, control of capital movements, economy policy, economies of Latin America

\section{AUTEUR}

\section{ANDRÉ CARTAPANIS}

Né à Marseille en 1952, André Cartapanis est Docteur d'État en sciences économiques et il est aujourd'hui professeur d'économie et de finances internationales à Sciences Po Aix-en-Provence, tout en étant chercheur au GREDEG, UMR 6227, CNRS-Université de Nice Sophia Antipolis, et au CHERPA, EA 4261 de l'IEP d'Aix-en-Provence. Ses publications les plus récentes portent sur les facteurs de déclenchement des crises financières et les questions de gouvernance financière internationale. Il a publié une vingtaine d'ouvrages (Transnational Corporations and Endogenous Development ; Instabilité des taux de change et déséquilibres internationaux; Turbulences et spéculations dans l'économie mondiale; Politique économique: fondements théoriques; Les marchés financiers internationaux; Regional Currency Areas in Financial Globalization...), plus de 80 articles dans des revues spécialisées ou des ouvrages collectifs (Revue Économique, Revue d'Économie politique, Revue de l'OFCE, Économie Internationale, Revue d'Économie Financière, Review of International Economics, Review of International Political Economy...), et il a contribué à de nombreux rapports pour le 
compte d'organismes nationaux ou internationaux (Commission Européenne, UNESCO, Banque de France, Conseil d'Analyse Économique...). Directeur, pendant dix ans, du Centre d'Économie et de Finances Internationales (CEFI), UMR 6126 du CNRS et de l'Université de la Méditerranée, il a assumé de nombreuses fonctions de direction ou d'évaluation au sein des instances universitaires : Doyen de la Faculté des Sciences Économiques d'Aix-Marseille, membre élu pendant douze ans du Conseil National des Universités, membre puis président du jury du concours d'agrégation de sciences économiques... Il est aujourd'hui responsable du master d'Affaires internationales de Sciences Po Aix-en-Provence. Membre du Cercle des économistes depuis 2006, il a été président de l'Association Française de Science Economique en 2009-2010. andre.cartapanisatsciencespo-aix.fr 